\title{
Glycothermally Synthesized Carbon Dots with Narrow-Bandwidth and Color-Tunable Solvatochromic Fluorescence for Wide-Color- Gamut Displays
}

Taishu Yoshinaga, Momoka Shinoda, Yoshiki Iso,* Tetsuhiko Isobe,* Akihiro Ogura, and Ken-ichi Takao

Cite This: ACS Omega 2021, 6, 1741-1750

Read Online

ABSTRACT: Fluorescent carbon dots (CDs) represent a Heated in an open system promising eco-friendly next-generation phosphor. However, most CDs exhibit broad photoluminescence (PL) spectra [full width at half-maximum (fwhm) over $60 \mathrm{~nm}$ ]; few works on CDs with sharp PL spectra (fwhm less than $40 \mathrm{~nm}$ ) have been reported. In addition, their syntheses and color tuning require harsh conditions of high temperatures, long reaction times, and high pressures with catalysts. Here, we successfully prepared narrow-bandwidth emissive CDs (fwhm of $27-40 \mathrm{~nm}$ ) from phloroglucinol in a glycol solvent of 1,2 -pentanediol at temperatures as low as $180^{\circ} \mathrm{C}$

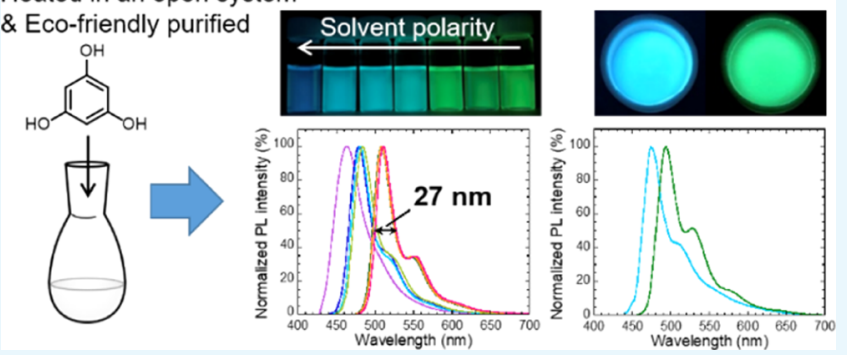
for a reaction duration of as short as $6 \mathrm{~h}$ under ambient conditions without any catalysts via an open reaction system in which dehydration and condensation reactions among phloroglucinol molecules were enhanced. We shifted the emission peak from 463 to $511 \mathrm{~nm}$ by selecting seven kinds of solvents with different polarities, that is, emission colors could be tuned from blue to green by taking advantage of fluorescence solvatochromism. The CD-dispersed polymer films showed a similar solvatochromic behavior and sharp PL spectra, verifying the feasibility of applying the CDs to displays with a wide color gamut.

\section{INTRODUCTION}

Phosphors are used in a wide variety of applications, such as lighting, displays, solar cells, and medical probes that are vital for society. Fluorescent carbon nanoparticles, called carbon dots (CDs), were discovered in 2004 and have received a great deal of attention as next-generation phosphors. ${ }^{1-10}$ CDs can be produced from a variety of organic materials and possess robust graphite structures, contributing to their environmental friendliness, high thermal stability, and high photostability. ${ }^{4,8,9}$ Because of these favorable characteristics, CDs are expected to serve as alternatives to quantum dots (QDs) of $\mathrm{CdSe} / \mathrm{ZnS}$ and $\mathrm{CsPbX}_{3}(\mathrm{X}=\mathrm{Cl}, \mathrm{Br}$, and $\mathrm{I})$ containing the toxic elements $\mathrm{Cd}$ and $\mathrm{Pb}$ and organic dyes. $\mathrm{CDs}$ have been applied in bioimaging, ${ }^{11,12}$ sensors for heavy metals, ${ }^{13,14}$ thermal sensors, ${ }^{14,15}$ and LEDs. ${ }^{16,17}$

The photoluminescence (PL) properties of CDs have been improved through the scrutiny of organic materials and the optimization of synthesis conditions and purification processes. Accordingly, the preparation of multicolor CDs with high PL quantum yields (QYs) has been reported. ${ }^{9,18,19}$ However, the PL spectra of CDs are usually broad, and their full widths at half-maximum (fwhms) exceed $60 \mathrm{~nm},{ }^{16,20,21}$ resulting in low emission color purity. The emission color purity is higher when the distribution of emission wavelengths, that is, the emission peak width, is narrower. There have been remarkably few reports on visible light-emissive CDs with fwhms less than 40 nm (Table 1). In contrast, QDs exhibit high color purity, with fwhms less than $30 \mathrm{~nm}$ (Table S1). To apply CDs to widecolor-gamut displays, it is desired to improve their color purity in the visible region.

The excitation-dependent broad-bandwidth emissive CDs have been produced through the pyrolysis and carbonization of organic materials. They contain different particle sizes and a variety of surface functional groups; ${ }^{10,19,29}$ therefore, they have diverse energy gaps and surface trap levels depending on their particle size distribution and surface functional groups, respectively, resulting in excitation-dependent broad-bandwidth emission. ${ }^{10,19,29}$

In recent years, excitation-independent emissive CDs have been prepared through polymerization of aromatic organic molecules such as phloroglucinol $(\mathrm{Ph})$ and phenylenediamine. $^{25,26,30}$ For example, Yuan et al. reported that blue-

Received: December 9, 2020

Accepted: December 29, 2020

Published: January 7, 2021 
Table 1. Previous Reports of Emission Peak Wavelengths $\left(\lambda_{\mathrm{em}}\right)$, fwhms, and Photoluminescence Quantum Yields of NarrowBandwidth Emissive CDs

\begin{tabular}{|c|c|c|c|c|c|c|}
\hline carbon source & synthetic method & $\begin{array}{l}\text { synthetic } \\
\text { conditions }\end{array}$ & $\lambda_{\mathrm{em}}(\mathrm{nm})$ & $\begin{array}{l}\text { fwhm } \\
(\mathrm{nm})\end{array}$ & $\begin{array}{l}\text { PLQY } \\
(\%)\end{array}$ & refs \\
\hline ethylene glycol & $\begin{array}{l}\text { heated in } \mathrm{H}_{2} \mathrm{SO}_{4} \text { at } 80{ }^{\circ} \mathrm{C} \text {, and then stirring for } 30 \mathrm{~min} \\
\text { deionized water }\end{array}$ & in after injecting & 519 & 38 & 63 & 22 \\
\hline formamide and glutathione & microwave solvothermal & $160{ }^{\circ} \mathrm{C}, 1 \mathrm{~h}$ & 683 & 30 & 17 & 23 \\
\hline 1,4-diamino-naphthalene & solvothermal & $200{ }^{\circ} \mathrm{C}, 12 \mathrm{~h}$ & 581 & 30 & 82 & 24 \\
\hline \multirow{4}{*}{ phloroglucinol } & \multirow[t]{2}{*}{ solvothermal } & $200{ }^{\circ} \mathrm{C}, 9 \mathrm{~h}$ & 472 & 30 & 66 & \multirow[t]{4}{*}{25} \\
\hline & & $200{ }^{\circ} \mathrm{C}, 24 \mathrm{~h}$ & 507 & 29 & 72 & \\
\hline & \multirow{2}{*}{ solvothermal with $\mathrm{HCl}$ or $\mathrm{H}_{2} \mathrm{SO}_{4}$} & $200^{\circ} \mathrm{C}, 2 \mathrm{~h}$ & 538 & 30 & 62 & \\
\hline & & $200{ }^{\circ} \mathrm{C}, 5 \mathrm{~h}$ & 598 & 30 & 54 & \\
\hline \multirow[t]{2}{*}{ resorcinol } & \multirow[t]{2}{*}{ solvothermal } & $200{ }^{\circ} \mathrm{C}, 4 \mathrm{~h}$ & 520 & 31 & 75 & \multirow[t]{2}{*}{26} \\
\hline & & $200{ }^{\circ} \mathrm{C}, 7 \mathrm{~h}$ & 610 & 33 & 72 & \\
\hline diaminonaphthalene and citric acid & $\begin{array}{l}\text { solvothermal at } 160{ }^{\circ} \mathrm{C} \text { for } 6 \mathrm{~h} \text {, and then } 200{ }^{\circ} \mathrm{C} \text { for } 1 \\
\mathrm{NH}_{3} \text { (aq) and } \mathrm{N}_{2} \mathrm{H}_{4} \text { hydrate for surface amination }\end{array}$ & $\mathrm{h}$ after adding & 433 & 35 & $70 \pm 10$ & 27 \\
\hline tris(4-amino-phenyl)amine & $\begin{array}{l}\text { solvothermal with tert-butyl hydroperoxide and } \\
\mathrm{HCl}\end{array}$ & $130{ }^{\circ} \mathrm{C}, 2 \mathrm{~h}$ & $615 \pm 2$ & $27 \pm 1$ & $84 \pm 5$ & 28 \\
\hline \multirow[t]{2}{*}{ phloroglucinol } & \multirow[t]{2}{*}{ heated in an open system } & \multirow[t]{2}{*}{$180^{\circ} \mathrm{C}, 6 \mathrm{~h}$} & 481 & 30 & 51 & \multirow[t]{2}{*}{ this work } \\
\hline & & & 511 & 27 & 48 & \\
\hline
\end{tabular}

Scheme 1. Schematic Illustration of the Synthetic and Color Tuning Strategy for Ph-CDs

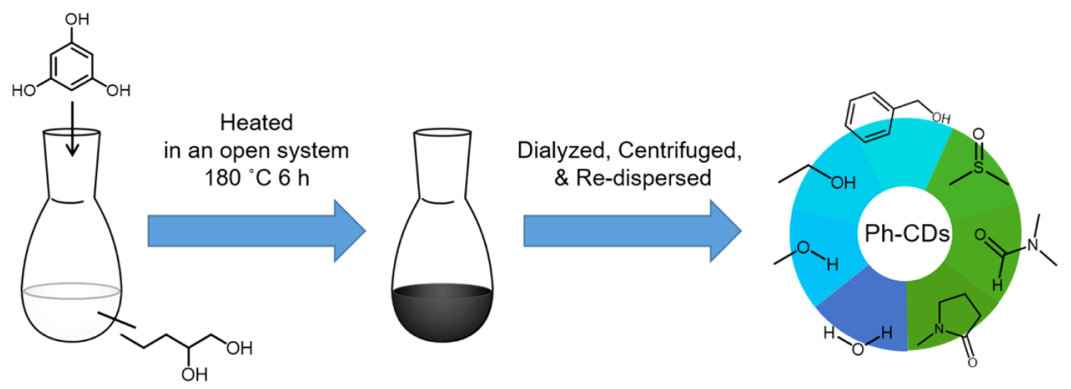

emitting CDs with an emission spectrum fwhm of $30 \mathrm{~nm}$ were produced via dehydration and condensation reactions between the $\mathrm{OH}$ groups of $\mathrm{Ph}$ molecules upon solvothermal treatment of a $\mathrm{Ph}$ ethanol solution at $200{ }^{\circ} \mathrm{C}$ for $9 \mathrm{~h}$ (Scheme S1). ${ }^{25}$ These authors performed silica column chromatography to purify the CDs and used the harmful substances dichloromethane and petroleum ether in the purification. ${ }^{25,26}$ The narrow-bandwidth emission originated from the triangular graphene structure of the CDs with a uniform size and homogeneous surface hydroxy groups. They demonstrated that the size of the $\pi$-conjugated graphene structure was increased through the dehydration and condensation reactions, resulting in a reduction in the energy gap between the highest occupied molecular orbital (HOMO) and the lowest unoccupied molecular orbital (LUMO) and thus leading to a change from blue emission to green and red emission. However, to increase the size of the structure to achieve longer emission wavelengths, a longer heating duration or the use of a dehydration catalyst involving additional purifications was required. $^{25,26}$

To prepare narrow-bandwidth emissive CDs at lower temperatures, with a shorter heating duration, and without high pressure and any catalysts, we focused on designing an open reaction system using a high-boiling solvent rather than the closed reaction system in an autoclave used in the previous reports (Table 1), because the dehydration and condensation reactions are expected to be promoted by removing water from the system. Actually, we demonstrated that CDs with higher photoluminescence quantum yields (PLQYs) were produced when these reactions were performed in an open system with a nonaqueous solvent possessing a high boiling point than when the reactions were performed in a closed system with an aqueous solvent. ${ }^{31}$ In a previous report, however, CDs prepared from $\mathrm{Ph}$ in an open system using a high-boiling solvent of ethylene glycol did not show high-quality fluorescence; their PLQY was $1 \% .^{32}$

The emission color can be controlled by tuning the CD size due to the quantum size effect, as mentioned above. ${ }^{25,26}$ In the present study, we focused on fluorescence solvatochromism as another color-controllable strategy because we can realize different emission colors using only one size of CDs and avoid the increase in the heating duration and the use of a dehydration catalyst like $\mathrm{H}_{2} \mathrm{SO}_{4}$ involving additional purifications. Recently, it has been reported that the emission wavelengths of phenylenediamine-derived CDs depend on the polarities of solvents and polymers. ${ }^{1,30,33-35}$ This fluorescence solvatochromism is ascribed to the difference in the dipole moments in the ground state and the excited state; the emission wavelength shifts with the change in polarity of the dispersion media. ${ }^{30,33-37}$ Hence, we can readily tune the desired emission wavelength through selection of the dispersion media. However, to our knowledge, the fluorescence solvatochromism of narrow-bandwidth emissive CDs listed in Table 1 has not yet been reported.

In the present study, we successfully established a novel atmospheric glycothermal synthesis of Ph-derived $\mathrm{CDs}$ (PhCDs) whose fwhms of emission peaks were $27-40 \mathrm{~nm}$. In this method, $\mathrm{Ph}$ was dissolved in a glycol solvent of 1,2-pentanediol possessing a boiling point of $207^{\circ} \mathrm{C}^{38}$ and heated at $180^{\circ} \mathrm{C}$ for $6 \mathrm{~h}$ in an open system under ambient conditions without a 
reflux unit, as illustrated in Scheme 1. The obtained dispersion was purified by the facile and eco-friendly procedure of dialysis and centrifugation using ultrapure water to yield $\mathrm{Ph}-\mathrm{CD}$ powders. We dispersed Ph-CD powders in solvents and polymers with different polarities and investigated their fluorescence solvatochromism (Scheme 1) and the feasibility of applying $\mathrm{Ph}-\mathrm{CD}$ s to wide-color-gamut displays.

\section{RESULTS AND DISCUSSION}

Particulate Properties, Chemical Bonding States, and Elemental Compositions of Ph-CDs. Figure la shows the
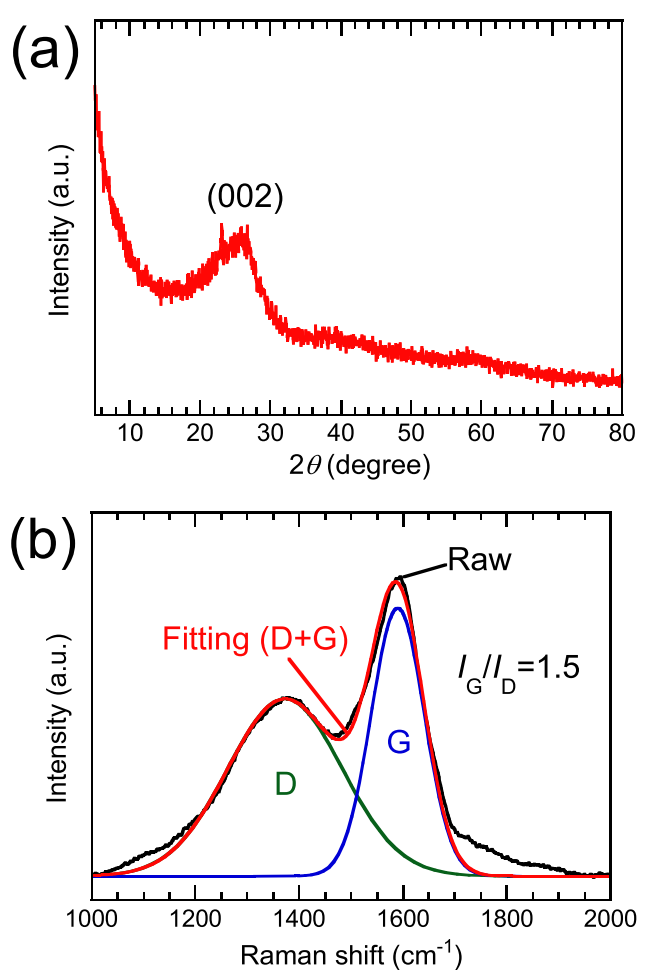

Figure 1. (a) XRD profile and (b) Raman spectrum of Ph-CDs.

X-ray diffraction (XRD) profile of the $\mathrm{Ph}-\mathrm{CD}$ powder. A broad diffraction peak was centered approximately $26^{\circ}$, which matches the peak of the (002) plane of graphite. Graphite is composed of stacked graphene layers in the $c$-axis orientation through $\pi-\pi$ interactions of van der Waals forces. ${ }^{39}$ The lattice spacing of the (002) plane matches the spacing between the graphene layers of $0.33 \mathrm{~nm} .{ }^{40}$ Numerous crystalline peaks of the $\mathrm{Ph}$ powder were not observed in the XRD profile of the $\mathrm{Ph}-\mathrm{CD}$ powder (see Figure S1). Therefore, we concluded that the graphite structure of the obtained $\mathrm{Ph}-\mathrm{CDs}$ was composed of stacked graphene in the $c$-axis orientation through $\pi-\pi$ interactions.

Figure $1 \mathrm{~b}$ depicts the Raman spectrum of the $\mathrm{Ph}-\mathrm{CD}$ powder. The $\mathrm{D}$ band, which corresponds to the disorder and defects of the graphite structure, and the G band, which corresponds to the in-plane motion of carbon atoms, were observed at 1370 and $1590 \mathrm{~cm}^{-1}$, respectively. ${ }^{41}$ The ordered structure of the $\mathrm{Ph}-\mathrm{CD}$ s can be determined by the intensity ratio of these $\mathrm{D}$ and $\mathrm{G}$ bands $\left(I_{\mathrm{G}} / I_{\mathrm{D}}\right){ }^{42} I_{\mathrm{G}} / I_{\mathrm{D}}$ of Ph-CDs was 1.5 (Table S2), which was higher than $I_{\mathrm{G}} / I_{\mathrm{D}}$ of the previously reported CDs, $1.0,{ }^{43}$ indicating the high crystallinity of the graphite structure of $\mathrm{Ph}-\mathrm{CDs}$.
The transmission electron microscopy (TEM) image of PhCDs is shown in Figure 2. Nanoparticles of $\sim 2 \mathrm{~nm}$ in size were

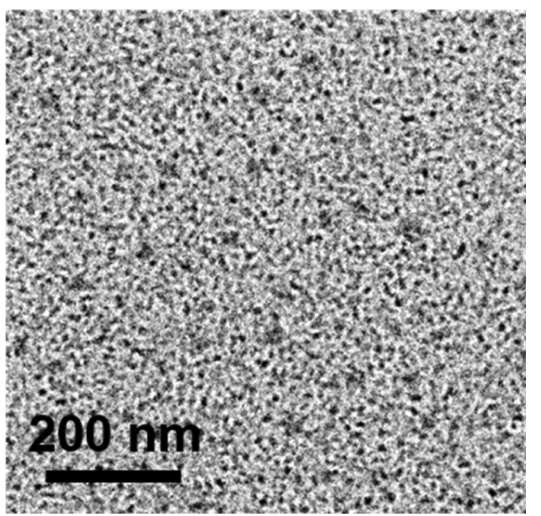

Figure 2. TEM image of Ph-CDs.

observed in this image. As shown in Figure S2, the lattice fringes of the triangular structure with a size of approximately 1-2 $\mathrm{nm}$ were observed in the high-resolution TEM image. According to the fast Fourier transform (FFT) pattern (Figure S2), the spacing of the lattice fringe was evaluated to be 0.24 $\mathrm{nm}$, which corresponds to the spacing of the (1120) plane of graphite. $^{44}$

Figure 3a illustrates the Fourier-transform infrared (FT-IR) spectrum of the $\mathrm{Ph}-\mathrm{CD}$ powder. The details of the peak
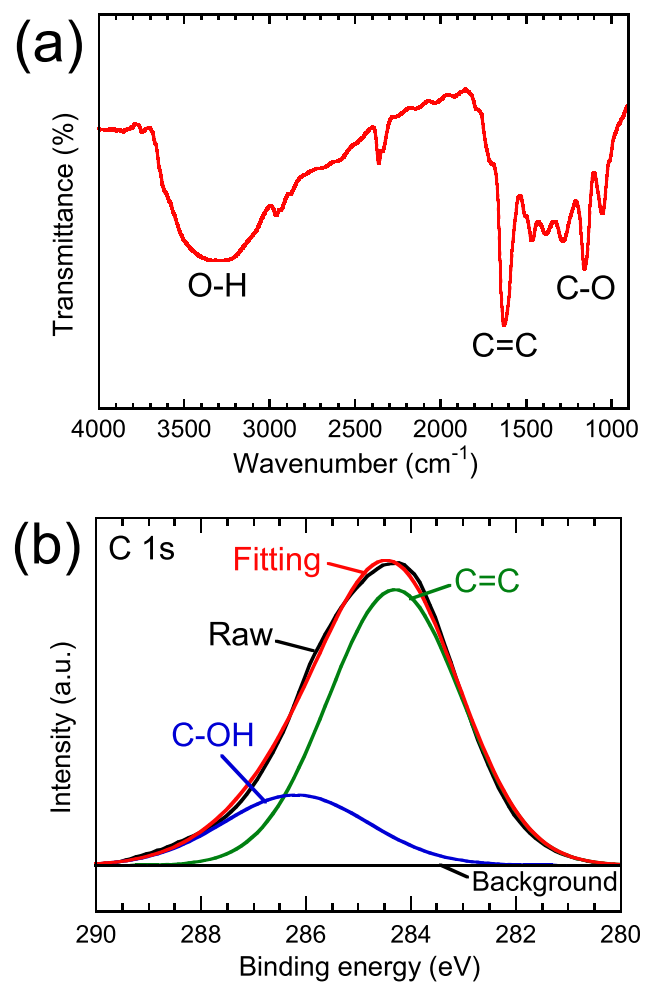

Figure 3. (a) FT-IR and (b) C 1s XPS spectra of Ph-CDs.

assignments are summarized in Figure S3 and Table S3. The peaks observed at $3100-3700,1630$, and $1158 \mathrm{~cm}^{-1}$ were assigned to the stretching vibrations of $\mathrm{O}-\mathrm{H}, \mathrm{C}=\mathrm{C}$, and $\mathrm{C}-\mathrm{O}$ bonds, respectively. ${ }^{45,46}$ The characteristic peak at $1068 \mathrm{~cm}^{-1}$ was observed in the FT-IR spectrum of the synthetic solvent of 1,2-pentanediol (Figure S3) and is assigned to the asymmetric 
stretching vibration of $\mathrm{C}-\mathrm{C}-\mathrm{O}$ bonds. ${ }^{45,46}$ This peak was not observed in the spectrum of the $\mathrm{Ph}-\mathrm{CD}$ powder, revealing that the solvent molecule did not bind to the edge of Ph-CDs.

The peaks of $\mathrm{C} 1 \mathrm{~s}$ and $\mathrm{O} 1 \mathrm{~s}$ were observed in the X-ray photoelectron spectroscopy (XPS) survey scan of the Ph-CD powder (Figure S4). Figures $3 \mathrm{~b}$ and $\mathrm{S} 4$ show the highresolution scans of the $\mathrm{C} 1 \mathrm{~s}$ and $\mathrm{O} 1 \mathrm{~s}$ regions, respectively. The $\mathrm{C} 1 \mathrm{~s}$ spectrum was fitted by two peaks, corresponding to C$\mathrm{OH}(286.2 \mathrm{eV})$ and $\mathrm{C}=\mathrm{C}$ bonds $(284.3 \mathrm{eV}){ }^{32,47}$ The peak observed at $532.5 \mathrm{eV}$ in the $\mathrm{O} 1 \mathrm{~s}$ spectrum was assigned to $\mathrm{C}-$ $\mathrm{OH}$ bonds. ${ }^{32}$ The presence of these bonds is consistent with the FT-IR results.

As shown in Figure S5, hydrogen signals from aromatic rings and hydroxy groups were detected at ca. 6.7 ppm and ca. 5.8 ppm in the ${ }^{1} \mathrm{H}$ nuclear magnetic resonance (NMR) spectrum (DMSO- $\left.d_{6}, \mathrm{ppm}\right)$ of the Ph-CDs, respectively. ${ }^{48}$ The chemical shifts of the hydrogen signals from aromatic rings were noticeably different between the $\mathrm{Ph}-\mathrm{CD}$ s and $\mathrm{Ph}$. The signal was detected at ca. $5.6 \mathrm{ppm}$ in the ${ }^{1} \mathrm{H}$ NMR spectrum (DMSO- $d_{6}, \mathrm{ppm}$ ) of $\mathrm{Ph}^{48}$ The difference of their chemical shifts indicates that the aromatic structure of the prepared $\mathrm{Ph}$ CDs was completely different from that of $\mathrm{Ph}$.

The above-mentioned results confirm that the prepared $\mathrm{Ph}$ CDs contain the ordered graphite structure of the $\pi$ conjugated systems and the homogeneous surface functional groups of $\mathrm{OH}$.

As illustrated in Table 2, the proportions of $\mathrm{C}-\mathrm{OH}$ and $\mathrm{C}=$ $\mathrm{C}$ bonds were calculated from the areas of the fitted $\mathrm{C}$ 1s XPS

Table 2. Proportions of Different Bonds Calculated from Fitting of the C 1s XPS of Ph-CDs and Ph

\begin{tabular}{lcc} 
sample & $\mathrm{C}-\mathrm{OH}(\%)$ & $\mathrm{C}=\mathrm{C}(\%)$ \\
$\mathrm{Ph}-\mathrm{CDs}$ & 21.6 & 78.4 \\
$\mathrm{Ph}$ & 39.8 & 60.2 \\
\hline
\end{tabular}

peaks (Figures $3 \mathrm{~b}$ and $\mathrm{S} 4$ ). The proportion of $\mathrm{C}-\mathrm{OH}$ bonds in the $\mathrm{Ph}-\mathrm{CD}$ powder was $21.6 \%$, which was lower than that in the $\mathrm{Ph}$ powder $(39.8 \%)$. The proportion of $\mathrm{C}=\mathrm{C}$ bonds in the $\mathrm{Ph}-\mathrm{CD}$ powder was $78.4 \%$, which was higher than that in the $\mathrm{Ph}$ powder $(60.2 \%)$. These results indicate that dehydration and condensation reactions between $\mathrm{Ph}$ molecules were facilitated in the synthetic process, implying the expansion of the $\pi$-conjugated structures, as shown in Scheme S1.

Table $\mathrm{S} 4$ shows the proportions of $\mathrm{C}, \mathrm{H}$, and $\mathrm{O}$ in the $\mathrm{Ph}$ $\mathrm{CD}$ powder, as determined by elemental analysis. Because the amount of $\mathrm{C}$ is constant regardless of the promotion of the dehydration and condensation reactions between $\mathrm{Ph}$ molecules, we calculated the $\mathrm{H} / \mathrm{C}$ and $\mathrm{O} / \mathrm{C}$ molar ratios from the elemental proportions in Table S4 (see Table S5). The H/C and $\mathrm{O} / \mathrm{C}$ ratios of the $\mathrm{Ph}-\mathrm{CDs}$ were lower than those of $\mathrm{Ph}$, indicating the progress of the dehydration and condensation reactions in the synthetic process.

Optical Properties of Ph-CDs Dispersed in Solvents with Different Polarities. $\mathrm{Ph}-\mathrm{CD}$ powders were dispersed in seven kinds of solvents with different polarities, water, methanol, ethanol, benzyl alcohol, dimethyl sulfoxide (DMSO), N,N-dimethylformamide (DMF), and 1-methyl-2pyrrolidone (MP), and these dispersions were transparent under white light, as shown in Figure 4.

This high dispersibility is attributed to the structure of the $\mathrm{Ph}$-CDs, possessing a hydrophobic $\pi$-conjugated system and hydrophilic $\mathrm{OH}$ groups at the edge. The UV-vis spectra of the

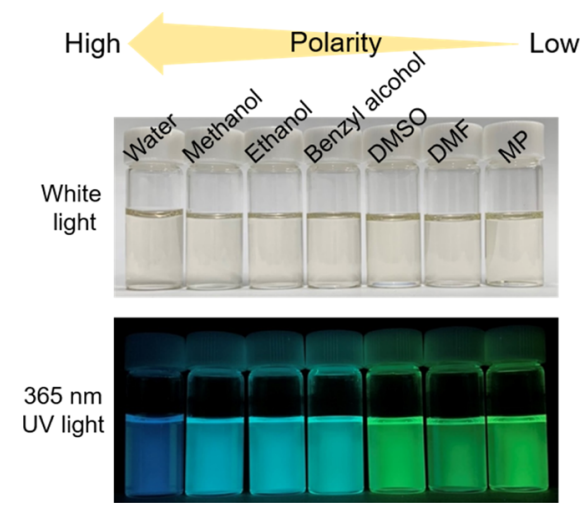

Figure 4. Photographs of $\mathrm{Ph}-\mathrm{CD}$ dispersions under white light and $365 \mathrm{~nm}$ UV light.

$\mathrm{Ph}-\mathrm{CD}$ dispersions (Figure S6) showed a blueshift of the absorption peak with an increase in the solvent polarity. The emission color of these dispersions changed from green to blue under UV light as the solvent polarity increased, as shown in Figure 4. The peaks observed in these UV-vis, photoluminescence excitation (PLE), and PL spectra (Figures 5, S6, and $\mathrm{S} 7$ ) were assigned to the HOMO-LUMO transition [ground state $\left(\mathrm{S}_{0}\right)$-excited state $\left(\mathrm{S}_{1}\right)$ transition; see Figures S8 and S9]. ${ }^{25,49}$
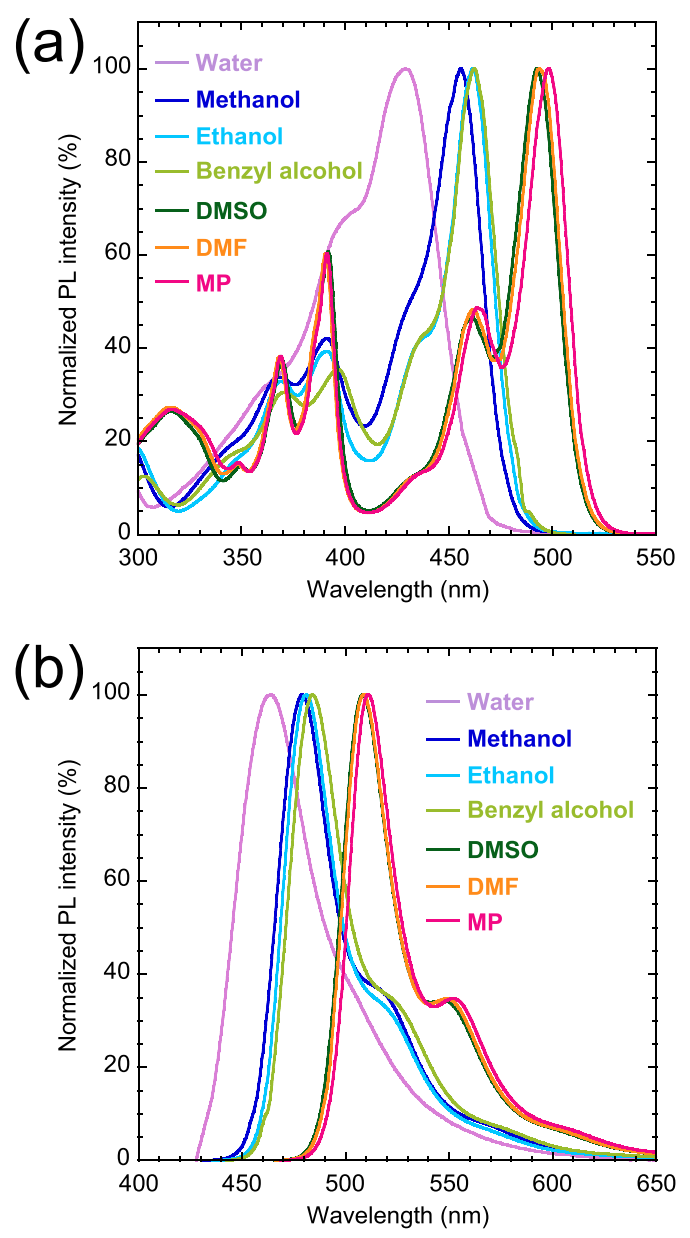

Figure 5. Normalized (a) PLE and (b) PL spectra of Ph-CD dispersions. 
The PL peaks were extraordinarily sharp, and the fwhms of the respective PL spectra (see Table S6) were $27-40 \mathrm{~nm}$. These values were narrower than those of previously reported excitation-dependent emissive CDs $(\geq 60 \mathrm{~nm})$ and comparable to those of QDs (Table S1).

The PLE and PL peaks shifted to shorter wavelengths as the solvent polarity increased. The emission energy linearly increased with the increase in each solvent polarity parameter $E_{\mathrm{T}}(30)$ (Figure 6 and Table 57 ), which reveals the negative fluorescence solvatochromism of $\mathrm{Ph}-\mathrm{CD}$ dispersions. ${ }^{36}$

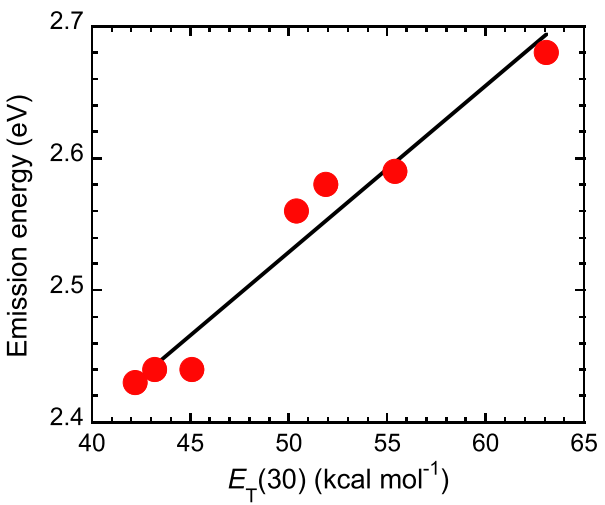

Figure 6. Relationship between the solvent polarity parameter $E_{\mathrm{T}}(30)$ and emission energy for $\mathrm{Ph}-\mathrm{CD}$ dispersions.

These results indicate that this negative fluorescence solvatochromism was attributed to the solvation effect rather than the localized effect of the formation of hydrogen bonds between the surface of the CDs and the solvent molecules. Hence, the ground state of $\mathrm{Ph}-\mathrm{CD}$ s stabilized due to solvation with increasing solvent polarity because the obtained $\mathrm{Ph}-\mathrm{CDs}$ had a larger dipole moment in the ground state rather than in the excited state (Figure 7 ). ${ }^{36}$

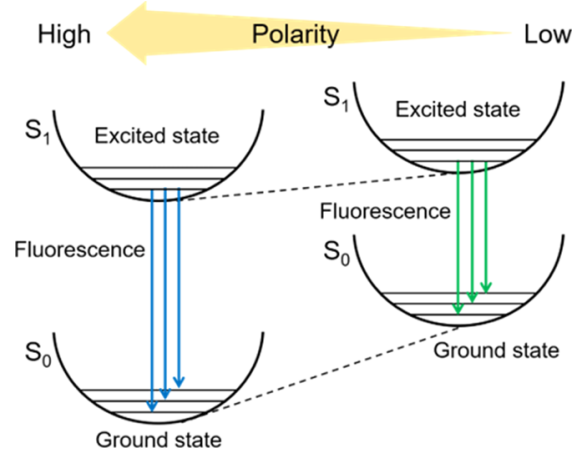

Figure 7. Proposed schematic illustration of the change in energy gaps of the Ph-CDs in different solvents.

Solvatochromism is not a common feature for phosphors. Inorganic phosphors doped with rare earth or transition metals, and semiconductor QDs do not exhibit solvatochromism. Although fluorescent organic molecules show solvatochromism, CDs do not always exhibit fluorescence solvatochromism. In fact, CDs prepared from D-glucose and L-cysteine in our previous reports did not show the solvatochromic behavior. ${ }^{31,50,51}$ Fluorescence solvatochromism for CDs has recently received a great deal of attention as their color tuning strategy and studied because CDs prepared from an aromatic compound of $p$-phenylenediamine showed the solvatochromic behavior. ${ }^{33,34,52}$ CDs prepared from aromatic compounds possessed $\pi$-conjugated systems, and their emission peaks shifted to longer wavelengths as the solvent polarity increased, that is, positive fluorescence solvatochromism. ${ }^{11,30,33-35,52,53}$ CDs prepared from nonaromatic compounds and possessing $\pi$ conjugated graphitic domains also exhibited positive fluorescence solvatochromism. ${ }^{54,55}$ This positive fluorescence solvatochromism is attributable to the reduction of the energy gaps of the CDs with increasing solvent polarity. ${ }^{30,35,36}$ In contrast, we found out that the emission peaks of the Ph-CDs shifted to shorter wavelengths as the solvent polarity increased, that is, negative fluorescence solvatochromism. Thus, the $\mathrm{Ph}$ CDs exhibited opposite shifts compared to the previously reported CDs.

All the UV-vis (Figure S6) and PLE spectra (Figures 5a and S7) of the $\mathrm{Ph}-\mathrm{CD}$ dispersions had shoulders and multiple peaks in the range from 350 to $550 \mathrm{~nm}$ and were fitted by six peaks (see Figure S8). These shoulders and peaks in the range from 400 to $550 \mathrm{~nm}$ were attributable to the transitions from the lowest vibrational level of the ground state $\left(S_{0}\right)$ to several vibrational levels of the excited state $\left(S_{1}\right)$ for the $\pi$-conjugated structure. $^{25,49}$ The peaks on the high energy side of their spectra ranging from 350 to $400 \mathrm{~nm}$ were attributable to the transitions from the lowest vibrational level of the ground state $\left(S_{0}\right)$ to several vibrational levels of the excited state $\left(S_{2}\right)$ for the $\pi$-conjugated structure. ${ }^{25,49}$ All the PL spectra of the Ph-CD dispersions had shoulders and were fitted by three peaks (see Figure S9). These shoulders were attributable to the transitions from the lowest vibrational level of the excited state $\left(S_{1}\right)$ to several vibrational levels of the ground state $\left(S_{0}\right)$ for the $\pi$ conjugated structure (see Figure S9). ${ }^{25,49}$ Figure S10 shows the respective PL spectra of the $\mathrm{Ph}-\mathrm{CD}$ dispersions as a function of emission energy. The respective fwhms are shown in Table S6. The fwhm increased with the increase in each solvent polarity parameter $E_{\mathrm{T}}(30)$ (Figure S11). This relationship was similar to that between the solvent polarity parameter $E_{\mathrm{T}}(30)$ and the emission energy (Figure 6). This indicates that the change in the fwhms can be attributed to the solvation effect. This change resulted in the change in the intensities of the shoulders of the PL spectra and their shape.

While Yuan et al. produced blue- and green-emitting CDs with fwhms of 30 and $29 \mathrm{~nm}$, respectively, from $\mathrm{Ph}$ under high pressure using an autoclave at $200{ }^{\circ} \mathrm{C}$ by changing the heating duration from 9 to $24 \mathrm{~h}$, followed by column chromatography purification using hazardous dichloromethane, ${ }^{25}$ we successfully prepared blue- and green-emitting $\mathrm{Ph}-\mathrm{CDs}$ with fwhms of 30 and $27 \mathrm{~nm}$, respectively, in an open system under ambient conditions at temperatures as low as $180{ }^{\circ} \mathrm{C}$ and reaction times as short as $6 \mathrm{~h}$, followed by facile and eco-friendly dialysis purifications using ultrapure water and redispersion in solvents by taking advantage of fluorescence solvatochromism. The PLQYs of both the blue and green emissions for the ethanol and DMSO dispersions of Ph-CDs were 51\% (Table S6 and Figure S12). Hence, the effective preparation of $\mathrm{Ph}-\mathrm{CDs}$ with multicolor narrow bandwidth emissions and high PLQYs was established. This novel type of atmospheric glycothermal method can also contribute to the mass production of narrowbandwidth emissive CDs because this open reaction system does not depend on the volume of a container and pressure to produce the CDs compared with the closed system using an autoclave (Table 1). 
The PL peak wavelengths for all the dispersions of $\mathrm{Ph}-\mathrm{CD}$ kept almost constant, regardless of the excitation wavelengths (Figure S13). This result indicates that the emission of the prepared $\mathrm{Ph}-\mathrm{CDs}$ is independent of the excitation wavelength, in contrast to the excitation-dependent emission of the CDs prepared through the pyrolysis and carbonization of organic molecules. ${ }^{6,7,56}$ This excitation-independent emission of $\mathrm{Ph}$ CDs is ascribed to their ordered $\pi$-conjugated structures and homogeneous surface functional groups, as mentioned above. ${ }^{57}$ This agrees well with the results of PL decay curves of $\mathrm{Ph}-\mathrm{CD}$ dispersions (Figure S14), which were fitted with the monoexponential equation. The PL lifetimes were evaluated to be 3.4-4.8 ns (Table S8).

As shown in the chromaticity coordinates converted from the PL spectra of the Ph-CD dispersions (Figure 8 and Table

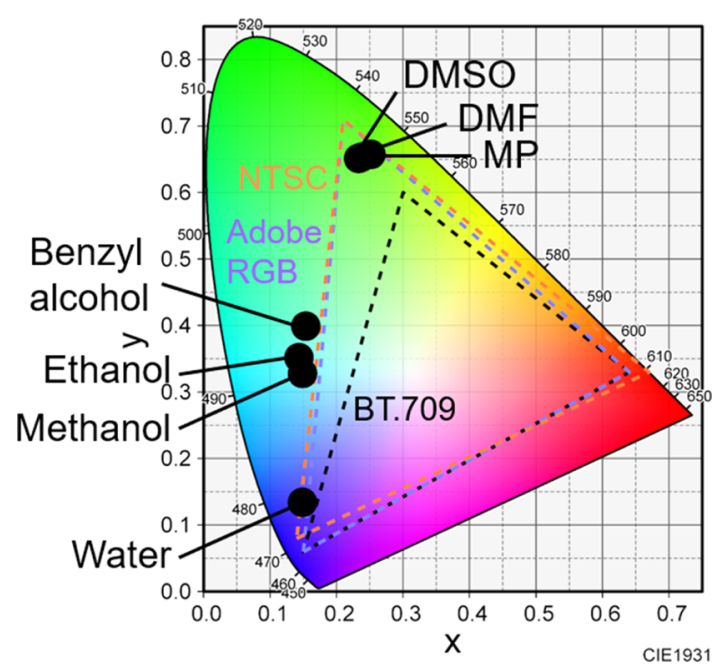

Figure 8. Chromaticity coordinates converted from the PL spectra of Ph-CD dispersions.

S9), these dispersions exhibited colors across the range of blue and green because of their narrow-bandwidth emissions and negative solvatochromic behavior. Notably, their color gamut exceeded the green vertex of the color system BT.709 of the conventional high-definition display and was close to that of the NTSC and the Adobe RGB.

Optical Properties of Ph-CDs Dispersed in Different Polymers. To investigate the feasibility of applying the prepared Ph-CDs to wide-color-gamut displays and LEDs, we formed $\mathrm{Ph}-\mathrm{CDs} /$ polymer composite films using polyvinyl alcohol (PVA) and polyvinyl pyrrolidone (PVP). The PhCDs were uniformly distributed in each polymer film, as shown in Figure 9. These $\mathrm{Ph}-\mathrm{CDs} / \mathrm{PVA}$ and $\mathrm{Ph}-\mathrm{CDs} / \mathrm{PVP}$ films exhibited blue and green emissions, respectively, under UV light (Figure 9).

The PLE peaks of the Ph-CDs/PVA and Ph-CDs/PVP films were located at 465 and $485 \mathrm{~nm}$, respectively, and their PL peaks were located at 474 and $494 \mathrm{~nm}$, respectively (Figures 10 and S15), indicating that those peak positions depend on the polymers. This color change is explained by the fluorescence solvatochromism attributed to the similarity in polarity between the polymers and the dispersions. ${ }^{33}$ These sharp PL peaks were similar to those of the dispersions; their fwhms were 33 and $35 \mathrm{~nm}$, respectively.

We attached both Ph-CDs/PVA and Ph-CDs/PVP films to a commercial UV LED to investigate the practical use of the $\mathrm{Ph}$ -

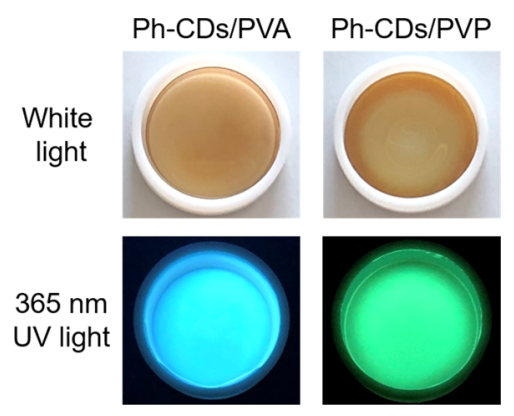

Figure 9. Photographs of Ph-CDs/PVA and Ph-CDs/PVP films under white light and $365 \mathrm{~nm}$ UV light.
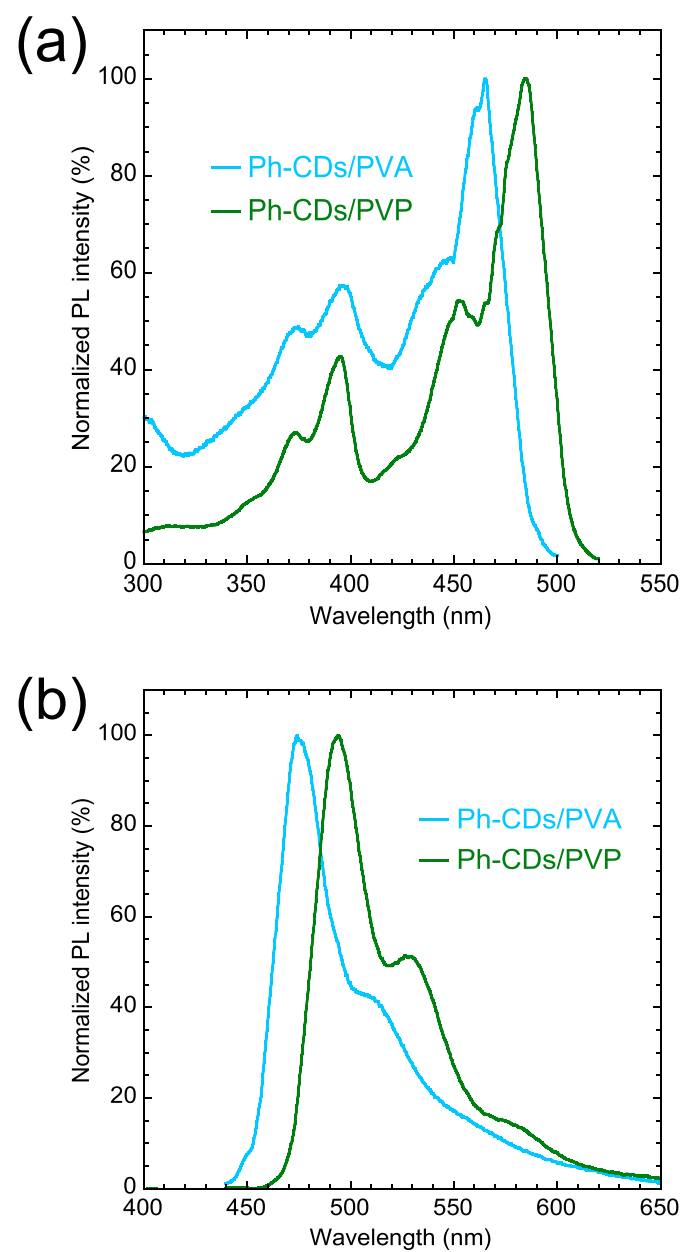

Figure 10. Normalized (a) PLE and (b) PL spectra of Ph-CDs/PVA and $\mathrm{Ph}-\mathrm{CDs} / \mathrm{PVP}$ films.

CDs for wide-color-gamut displays and LEDs, as shown in Figure 11. These Ph-CDs/PVA and Ph-CDs/PVP films converted the UV light to blue and green emissions, respectively, under white light. These results verify that the solvatochromic color-tuning strategy can design wavelength conversion films of desired emissive colors using polymers with appropriate polarities and the obtained Ph-CDs have substantial potential for practical application in wide-colorgamut displays and LEDs.

\section{CONCLUSIONS}

We successfully prepared narrow-bandwidth emissive Ph-CDs at temperatures as low as $180{ }^{\circ} \mathrm{C}$ for a reaction duration of as 


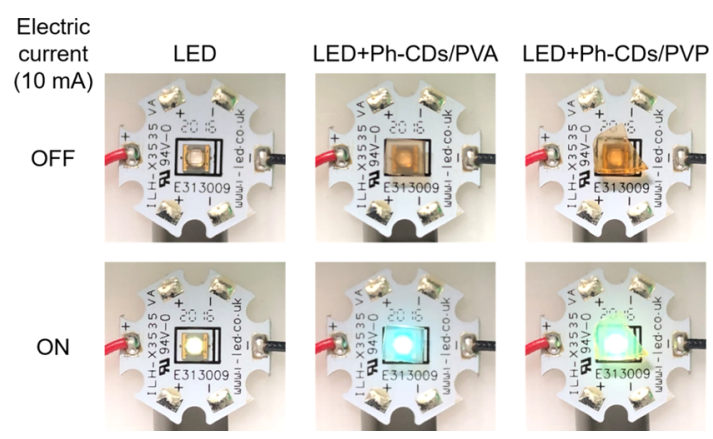

Figure 11. Photographs of the UV LED (Intelligent LED Solutions, ILH-XP01-S365-SC211-WIR200) with the Ph-CDs/polymer film under white light.

short as $6 \mathrm{~h}$ under ambient conditions without any catalysts via an open reaction system using a glycol solvent of 1,2pentanediol. The intermolecular dehydration and condensation reactions among $\mathrm{Ph}$ were facilitated in the open system, resulting in the ordered graphite structure and the homogeneous surface functional groups of $\mathrm{OH}$ of $\mathrm{Ph}-\mathrm{CD}$. $\mathrm{Ph}-\mathrm{CD}$ powders were dispersed in seven solvents with different polarities because of the hydrophobic $\pi$-conjugated system and hydrophilic $\mathrm{OH}$ groups. The emission peak of $\mathrm{Ph}-\mathrm{CD}$ s was readily tuned from 463 to $511 \mathrm{~nm}$ by taking advantage of negative fluorescence solvatochromism. They exhibited excitation-independent narrow-bandwidth blue and green emissions with respective fwhms of 30 and $27 \mathrm{~nm}$ and high PLQYs of up to $51 \%$. Ph-CD polymer composite films showed a similar solvatochromic behavior and narrow-bandwidth emissions. This novel atmospheric glycothermal preparation of narrow-bandwidth emissive CDs can contribute to their mass production and we verified the feasibility of applying them to wide-color-gamut displays and LEDs.

\section{EXPERIMENTAL SECTION}

Reagents. Ph (99\%), benzyl alcohol (99.0\%), MP (>99.0\%), and PVP $\left(M_{\mathrm{W}} 360,000\right)$ were purchased from FUJIFILM Wako Pure Chemical Industries. 1,2-pentanediol (>98.0\%) was purchased from Tokyo Chemical Industry. Methanol (99.8\%), ethanol (99.5\%), DMF (99.5\%), and DMSO (99.0\%) were purchased from Kanto Chemical Corporation. PVA (87-89\% hydrolyzed, $M_{\mathrm{W}} 85,000-$ $124,000)$ was purchased from Sigma-Aldrich. All reagents were used as received without further purification.

Preparation of Powders and Dispersions of Ph-CDs. $\mathrm{Ph}-\mathrm{CD}$ s were prepared via an effective method through the heating in an open reaction system using a high boiling solvent. $\mathrm{Ph}(2.0 \mathrm{~g})$ was added into a recovery flask $(100 \mathrm{~mL})$ containing the high boiling solvent of 1,2-pentanediol $(40 \mathrm{~mL})$ and dissolved in the solvent using ultrasonication. This solution was heated at $180{ }^{\circ} \mathrm{C}$ for $6 \mathrm{~h}$ with the use of an oil bath under ambient conditions and without a reflux unit. After cooling the flask to room temperature, the obtained suspension was dialyzed in ultrapure water ( 1 L) using a dialysis membrane (molecular weight cutoff of $3500 \mathrm{Da}$ : pore size of $1 \mathrm{~nm}$ ) for 2 days while stirring. In this dialysis, the ultrapure water was changed three times. During the purification process of this dialysis, precursors of Ph-CDs, unreacted materials, and 1,2-pentanediol molecules with the diameters less than $1 \mathrm{~nm}$ were removed. After adding ultrapure water $(30 \mathrm{~mL})$ into the dialyzed suspension $(10 \mathrm{~mL})$, this mixture was centrifuged at $\sim 16,000 \mathrm{~g}(13,000 \mathrm{rpm}$ using a rotor with a diameter of $10 \mathrm{~cm})$ for $5 \mathrm{~min}$. The supernatant solution was removed to collect the precipitate. After adding ultrapure water $(30 \mathrm{~mL})$ into the collected precipitate, the resulting mixture was centrifuged at the same rotation speed. The supernatant solution was removed to yield the precipitate. 1,2-Pentanediol was eliminated through this centrifugation process. The collected precipitate was dried using a rotary evaporator to yield $\mathrm{Ph}-\mathrm{CD}$ powder. The prepared Ph-CD powder was dispersed in water, methanol, ethanol, benzyl alcohol, DMSO, DMF, and MP to obtain the seven kinds of Ph-CD dispersions. The absorbance of each $\mathrm{Ph}-\mathrm{CD}$ dispersion was adjusted to be 0.05 at its each PLE peak wavelength for analysis.

Preparation of Ph-CDs/PVA Film. PVA (0.50 g) was added into ultrapure water $(4.5 \mathrm{~mL})$, stirred for $15 \mathrm{~min}$, and heated at $60{ }^{\circ} \mathrm{C}$ for $20 \mathrm{~min}$ under stirring. The $\mathrm{Ph}-\mathrm{CD}$ powder $(1.0 \mathrm{mg})$ was dispersed in ultrapure water $(4 \mathrm{~mL})$. This water dispersion of $\mathrm{Ph}-\mathrm{CDs}(1 \mathrm{~mL})$ was uniformly mixed with the prepared PVA aqueous solution $(4.5 \mathrm{~mL})$ under stirring and ultrasonication. The obtained mixture was poured into a polytetrafluoroethylene (PTFE) Petri plate $(30 \mathrm{~mm} \varphi \times 12$ $\mathrm{mmH}$ ) and dried for 3 days under ambient conditions in the dark to obtain the Ph-CDs/PVA film with a Ph-CD concentration of 0.05 wt $\%$.

Preparation of Ph-CDs/PVP Film. PVP ( $0.50 \mathrm{~g})$ was dissolved in ethanol $(4.5 \mathrm{~mL})$ using ultrasonication. The $\mathrm{Ph}$ CD powder $(1.0 \mathrm{mg})$ was dispersed in ethanol $(4 \mathrm{~mL})$. This ethanol dispersion of $\mathrm{Ph}-\mathrm{CDs}(1 \mathrm{~mL})$ was added into the prepared PVP ethanol solution $(4.5 \mathrm{~mL})$ and uniformly dispersed in the solution under stirring and ultrasonication. The obtained mixture was poured into the PTFE Petri plate and dried for 1 day under ambient conditions in the dark to obtain Ph-CDs/PVP film with a Ph-CD concentration of 0.05 wt \%.

Characterization. XRD profiles of $\mathrm{Ph}$ and $\mathrm{Ph}-\mathrm{CD}$ powder samples were measured on an X-ray diffractometer (Rigaku, Rint-2200) with a $\mathrm{Cu} \mathrm{K} \alpha$ radiation source and a monochromator. The Raman spectrum of the Ph-CD powder sample was recorded on a Raman microscope (Renishaw, in Via StreamLine) with a $532 \mathrm{~nm}$ laser. The Raman spectrum was fitted by two peaks using a software program (OriginLab Corp., Origin Pro 2019). The particle sizes and morphologies of $\mathrm{Ph}$-CDs were observed with a field-emission transmission electron microscope (FEI, Tecnai $\mathrm{G}^{2}$ ) at $200 \mathrm{kV}$. The sample for TEM observation was prepared by drying a drop of the concentrated $\mathrm{Ph}-\mathrm{CD}$ dispersion in ethanol on a copper grid covered with an ultrathin carbon-deposited film (Oken Shoji, HRC-C10) overnight. FT-IR absorption spectra of powder samples and 1,2-pentanediol in the pressed $\mathrm{KBr}$ disks were measured on a spectrometer (JASCO, FT/IR-4200). Chemical bonding states of powder samples were determined by XPS with an X-ray photoelectron spectroscopy instrument (JEOL, JPS-9010TR) using an $\mathrm{Al} \mathrm{K} \alpha$ radiation source. The peak of $\mathrm{C}$ $1 \mathrm{~s}$ at $284.4 \mathrm{eV}$ was used for the charge-up correction. ${ }^{1} \mathrm{H}$ NMR spectra were recorded at $500 \mathrm{MHz}$ on a spectrometer (JEOL, JNM-ECA500), and chemical shift was referenced internally to a residual DMSO signal at $2.49 \mathrm{ppm}$. The elemental composition of the Ph-CD powder was determined with an elemental analyzer (Elementar Analytical, Vario EL). The proportions of individual elements were qualified from the analysis of decomposed gas species in argon gas. UV-vis absorption spectra of $\mathrm{Ph}-\mathrm{CD}$ dispersions were measured with an UV/visible/near-infrared optical absorption spectrometer 
(JASCO, V-570). The net absorbance of each Ph-CD dispersion was calculated by subtracting the absorbance of each solvent from that of each Ph-CD dispersion. PLE and PL spectra of $\mathrm{Ph}-\mathrm{CD}$ dispersions were measured with a fluorescent spectrometer (JASCO, FP-6500). PLE and PL spectra of Ph$\mathrm{CD}$ films were measured with the fluorescent spectrometer equipped with an integrating sphere (JASCO, ISF-513); the excitation light was vertically irradiated onto the front side, and the emitted light was collected by the integrating sphere on the back side for detection. The spectral response was calibrated against a solution of rhodamine $\mathrm{B}$ in ethylene glycol $(5.5 \mathrm{~g}$ $\mathrm{L}^{-1}$ ) and a standard light source (JASCO, ESC-333). The PLQY of each Ph-CD dispersion was calculated relative to $95 \%$ of rhodamine 6G in ethanol. The absolute PLQY of each Ph$\mathrm{CD}$ dispersion was measured with a quantum efficiency measurement system (Otsuka Electronics, QE-2000-311C). $\mathrm{PL}$ decay curves of $\mathrm{Ph}-\mathrm{CD}$ dispersions were recorded on a fluorescence lifetime spectrometer (Hamamatsu Photonics, Quantaurus-Tau C11367), which was equipped with 405 and $470 \mathrm{~nm}$ LEDs as the light sources. These curves were fitted with the following monoexponential eq 1

$$
f(t)=A \exp (-t / \tau)
$$

where $f(t)$ is the PL intensity at time $t, A$ is the amplitude, and $\tau$ is the PL decay time.

\section{ASSOCIATED CONTENT}

\section{(s) Supporting Information}

The Supporting Information is available free of charge at https://pubs.acs.org/doi/10.1021/acsomega.0c05993.

Previous reports of QDs of CdSe/ZnS, CdSe/CdS/ZnS, $\mathrm{CdSe} / \mathrm{CdS}, \mathrm{Cd}_{1-x} \mathrm{Zn}_{x} \mathrm{~S} / \mathrm{ZnS}, \mathrm{InP} / \mathrm{ZnS}$, cesium lead halide perovskites $\left(\mathrm{CsPbX}_{3}, \mathrm{X}=\mathrm{Cl}, \mathrm{Br}\right.$, and $\left.\mathrm{I}\right)$, and organometal halide perovskites $\left(\mathrm{CH}_{3} \mathrm{NH}_{3} \mathrm{PbX}_{3}, \mathrm{X}=\mathrm{Cl}\right.$, $\mathrm{Br}$, and I); schematic illustration of the formation process of Ph-CDs; XRD profiles of $\mathrm{Ph}-\mathrm{CD}$ s and $\mathrm{Ph}$; intensities of the decomposed $\mathrm{D}\left(I_{\mathrm{D}}\right)$ and $\mathrm{G}\left(I_{\mathrm{G}}\right)$ and the calculated intensity ratio $\left(I_{\mathrm{G}} / I_{\mathrm{D}}\right)$; high-resolution TEM image and FFT pattern of Ph-CDs; FT-IR spectra of Ph$\mathrm{CDs}, \mathrm{Ph}$, and 1,2-pentanediol; assignment of FT-IR absorption peaks of Figure S3; XPS survey and highresolution scans of $\mathrm{Ph}-\mathrm{CDs}$ and $\mathrm{Ph} ;{ }^{1} \mathrm{H}$ NMR spectra of $\mathrm{Ph}-\mathrm{CDs}$ and $\mathrm{Ph}$; proportions of $\mathrm{C}, \mathrm{H}$, and $\mathrm{O}$ of $\mathrm{Ph}-\mathrm{CDs}$ determined by elemental analysis; elemental molar ratios of $\mathrm{Ph}-\mathrm{CDs}$ and $\mathrm{Ph}$ determined from Table S4; UV-vis spectra of Ph-CD dispersions; PLE and PL spectra of each Ph-CD dispersion; schematic illustration of the $S_{0}$ $\rightarrow S_{1}$ transitions from the ground state to the excited state including vibration levels, and the PLE spectrum of the $\mathrm{Ph}-\mathrm{CD}$ dispersion in MP and its peak fitting results; schematic illustration of the $S_{1} \rightarrow S_{0}$ transitions from the excited state to the ground state including vibration levels and the PL spectrum of the Ph-CD dispersion in ethanol and its peak fitting results; PLE and PL peak wavelengths of $\mathrm{Ph}-\mathrm{CD}$ dispersions, fwhms of their $\mathrm{PL}$ spectra, and their PLQYs; solvent polarity parameter $E_{\mathrm{T}}(30)$ values and emission peak wavelengths and energies for Ph-CD dispersions; normalized PL spectra of Ph-CD dispersions; relationship between the solvent polarity parameter $E_{\mathrm{T}}(30)$ and fwhms of the PL spectra of $\mathrm{Ph}-\mathrm{CD}$ dispersions; spectra of each solvent and $\mathrm{Ph}$ $\mathrm{CD}$ dispersion for evaluating the absolute PLQY in the regions of 400-900 and 400-700 nm; PL spectra of Ph$\mathrm{CD}$ dispersions, excited by different excitation wavelengths; $\mathrm{PL}$ decay curves of $\mathrm{Ph}-\mathrm{CD}$ dispersions; $\mathrm{PL}$ decay lifetimes of $\mathrm{Ph}-\mathrm{CD}$ dispersions; chromaticity coordinates converted from the PL spectra of Ph-CD dispersions; and PLE and PL spectra of Ph-CDs/PVA and $\mathrm{Ph}-\mathrm{CDs} / \mathrm{PVP}$ films (PDF)

\section{AUTHOR INFORMATION}

\section{Corresponding Authors}

Yoshiki Iso - Department of Applied Chemistry, Faculty of Science and Technology, Keio University, Yokohama 2238522, Japan; ำ orcid.org/0000-0001-7483-2828; Phone: +81 45566 1558; Email: iso@applc.keio.ac.jp; Fax: +81455661551

Tetsuhiko Isobe - Department of Applied Chemistry, Faculty of Science and Technology, Keio University, Yokohama 2238522, Japan; 이이이.org/0000-0002-0868-5425; Phone: +81 45566 1554; Email: isobe@applc.keio.ac.jp; Fax: +81455661551

\section{Authors}

Taishu Yoshinaga - Department of Applied Chemistry, Faculty of Science and Technology, Keio University, Yokohama 223-8522, Japan

Momoka Shinoda - Department of Applied Chemistry, Faculty of Science and Technology, Keio University, Yokohama 223-8522, Japan

Akihiro Ogura - Department of Applied Chemistry, Faculty of Science and Technology, Keio University, Yokohama 2238522, Japan; (1) orcid.org/0000-0002-4793-5706

Ken-ichi Takao - Department of Applied Chemistry, Faculty of Science and Technology, Keio University, Yokohama 2238522, Japan; 이이.org/0000-0001-8629-1002

Complete contact information is available at:

https://pubs.acs.org/10.1021/acsomega.0c05993

\section{Notes}

The authors declare no competing financial interest.

\section{ACKNOWLEDGMENTS}

This work was supported by the Japan Society for the Promotion of Science via KAKENHI grant number JP19K22236. T.Y. thanks the Japan Society for the Promotion of Science for the doctoral fellowship (DC2).

\section{REFERENCES}

(1) Xu, X.; Ray, R.; Gu, Y.; Ploehn, H. J.; Gearheart, L.; Raker, K.; Scrivens, W. A. Electrophoretic Analysis and Purification of Fluorescent Single-Walled Carbon Nanotube Fragments. J. Am. Chem. Soc. 2004, 126, 12736-12737.

(2) Sun, Y.-P.; Zhou, B.; Lin, Y.; Wang, W.; Fernando, K. A. S.; Pathak, P.; Meziani, M. J.; Harruff, B. A.; Wang, X.; Wang, H.; Luo, P. G.; Yang, H.; Kose, M. E.; Chen, B.; Veca, L. M.; Xie, S.-Y. QuantumSized Carbon Dots for Bright and Colorful Photoluminescence. J. Am. Chem. Soc. 2006, 128, 7756-7757.

(3) Liu, H.; Ye, T.; Mao, C. Fluorescent Carbon Nanoparticles Derived from Candle Soot. Angew. Chem., Int. Ed. 2007, 46, 64736475.

(4) Baker, S. N.; Baker, G. A. Luminescent Carbon Nanodots: Emergent Nanolights. Angew. Chem., Int. Ed. 2010, 49, 6726-6744.

(5) Pan, D.; Zhang, J.; Li, Z.; Wu, M. Hydrothermal Route for Cutting Graphene Sheets into Blue-Luminescent Graphene Quantum Dots. Adv. Mater. 2010, 22, 734-738. 
(6) Tang, L.; Ji, R.; Cao, X.; Lin, J.; Jiang, H.; Li, X.; Teng, K. S.; Luk, C. M.; Zeng, S.; Hao, J.; Lau, S. P. Deep Ultraviolet Photoluminescence of Water-Soluble Self-Passivated Graphene Quantum Dots. ACS Nano 2012, 6, 5102-5110.

(7) Zhu, S.; Meng, Q.; Wang, L.; Zhang, J.; Song, Y.; Jin, H.; Zhang, K.; Sun, H.; Wang, H.; Yang, B. Highly Photoluminescent Carbon Dots for Multicolor Patterning, Sensing, and Bioimaging. Angew. Chem., Int. Ed. 2013, 52, 3953-3957.

(8) Hola, K.; Zhang, Y.; Wang, Y.; Giannelis, E. P.; Zboril, R.; Rogach, A. L. Carbon Dots-Emerging Light Emitters for Bioimaging, Cancer Therapy and Optoelectronics. Nano Today 2014, 9, 590-603.

(9) Zheng, X. T.; Ananthanarayanan, A.; Luo, K. Q.; Chen, P. Glowing Graphene Quantum Dots and Carbon Dots: Properties, Syntheses, and Biological Applications. Small 2015, 11, 1620-1636.

(10) Ding, H.; Yu, S.-B.; Wei, J.-S.; Xiong, H.-M. Full-Color LightEmitting Carbon Dots with a Surface-State-Controlled Luminescence Mechanism. ACS Nano 2016, 10, 484-491.

(11) Xia, J.; Chen, S.; Zou, G.-Y.; Yu, Y.-L.; Wang, J.-H. Synthesis of Highly Stable Red-Emissive Carbon Polymer Dots by Modulated Polymerization: from the Mechanism to Application in Intracellular pH Imaging. Nanoscale 2018, 10, 22484-22492.

(12) Yang, S.-T.; Cao, L.; Luo, P. G.; Lu, F.; Wang, X.; Wang, H.; Meziani, M. J.; Liu, Y.; Qi, G.; Sun, Y.-P. Carbon Dots for Optical Imaging in Vivo. J. Am. Chem. Soc. 2009, 131, 11308-11309.

(13) Dang, D. K.; Sundaram, C.; Ngo, Y.-L. T.; Chung, J. S.; Kim, E. J.; Hur, S. H. One Pot Solid-State Synthesis of Highly Fluorescent N and S Co-Doped Carbon Dots and Its Use as Fluorescent Probe for $\mathrm{Ag}^{+}$Detection in Aqueous Solution. Sens. Actuators, B 2018, 255, 3284-3291.

(14) Cui, X.; Wang, Y.; Liu, J.; Yang, Q.; Zhang, B.; Gao, Y.; Wang, Y.; Lu, G. Dual Functional N- and S-Co-Doped Carbon Dots as the Sensor for Temperature and $\mathrm{Fe}^{3+}$ ions. Sens. Actuators, B 2017, 242, $1272-1280$

(15) Wang, C.; Xu, Z.; Cheng, H.; Lin, H.; Humphrey, M. G.; Zhang, C. A Hydrothermal Route to Water-Stable Luminescent Carbon Dots as Nanosensors for $\mathrm{pH}$ and Temperature. Carbon 2015, 82, 87-95.

(16) Yuan, F.; Wang, Z.; Li, X.; Li, Y.; Tan, Z. a.; Fan, L.; Yang, S. Bright Multicolor Bandgap Fluorescent Carbon Quantum Dots for Electroluminescent Light-Emitting Diodes. Adv. Mater. 2017, 29, 1604436.

(17) Miao, X.; Qu, D.; Yang, D.; Nie, B.; Zhao, Y.; Fan, H.; Sun, Z. Synthesis of Carbon Dots with Multiple Color Emission by Controlled Graphitization and Surface Functionalization. Adv. Mater. 2018, 30, 1704740.

(18) Du, Y.; Guo, S. Chemically Doped Fluorescent Carbon and Graphene Quantum Dots for Bioimaging, Sensor, Catalytic and Photoelectronic Applications. Nanoscale 2016, 8, 2532-2543.

(19) Mintz, K. J.; Zhou, Y.; Leblanc, R. M. Recent Development of Carbon Quantum Dots Regarding Their Optical Properties, Photoluminescence Mechanism, and Core Structure. Nanoscale 2019, 11, $4634-4652$.

(20) Xie, Z.; Wang, F.; Liu, C.-Y. Organic-Inorganic Hybrid Functional Carbon Dot Gel Glasses. Adv. Mater. 2012, 24, 17161721.

(21) Bao, L.; Liu, C.; Zhang, Z.-L.; Pang, D.-W. PhotoluminescenceTunable Carbon Nanodots: Surface-State Energy-Gap Tuning. Adv. Mater. 2015, 27, 1663-1667.

(22) Liu, Y.; Liu, C.-Y.; Zhang, Z.-Y. Graphitized Carbon Dots Emitting Strong Green Photoluminescence. J. Mater. Chem. C 2013, 1, 4902-4907.

(23) Pan, L.; Sun, S.; Zhang, L.; Jiang, K.; Lin, H. Near-Infrared Emissive Carbon Dots for Two-Photon Fluorescence Bioimaging. Nanoscale 2016, 8, 17350-17356.

(24) Han, Z.; Ni, Y.; Ren, J.; Zhang, W.; Wang, Y.; Xie, Z.; Zhou, S.; Yu, S. F. Highly Efficient and Ultra-Narrow Bandwidth Orange Emissive Carbon Dots for Microcavity Lasers. Nanoscale 2019, 11, 11577-11583.
(25) Yuan, F.; Yuan, T.; Sui, L.; Wang, Z.; Xi, Z.; Li, Y.; Li, X.; Fan, L.; Tan, Z.; Chen, A.; Jin, M.; Yang, S. Engineering Triangular Carbon Quantum Dots with Unprecedented Narrow Bandwidth Emission for Multicolored LEDs. Nat. Commun. 2018, 9, 2249.

(26) Yuan, F.; He, P.; Xi, Z.; Li, X.; Li, Y.; Zhong, H.; Fan, L.; Yang, S. Highly Efficient and Stable White LEDs Based on Pure Red Narrow Bandwidth Emission Triangular Carbon Quantum Dots for Wide-Color Gamut Backlight Displays. Nano Res. 2019, 12, 16691674.

(27) Yuan, F.; Wang, Y.-K.; Sharma, G.; Dong, Y.; Zheng, X.; Li, P.; Johnston, A.; Bappi, G.; Fan, J. Z.; Kung, H.; Chen, B.; Saidaminov, M. I.; Singh, K.; Voznyy, O.; Bakr, O. M.; Lu, Z.-H.; Sargent, E. H. Bright High-Colour-Purity Deep-Blue Carbon Dot Light-Emitting Diodes via Efficient Edge Amination. Nat. Photonics 2020, 14, 171176.

(28) Liu, Y.; Gou, H.; Huang, X.; Zhang, G.; Xi, K.; Jia, X. Rational Synthesis of Highly Efficient Ultra-Narrow Red-Emitting Carbon Quantum Dots for NIR-II Two-Photon Bioimaging. Nanoscale 2020, 12, 1589-1601.

(29) Dong, Y.; Pang, H.; Yang, H. B.; Guo, C.; Shao, J.; Chi, Y.; Li, C. M.; Yu, T. Carbon Based Dots Co-Doped with Nitrogen and Sulfur for High Quantum Yield and Excitation Independent Emission. Angew. Chem., Int. Ed. 2013, 52, 7800-7804.

(30) Sato, R.; Iso, Y.; Isobe, T. Fluorescence Solvatochromism of Carbon Dot Dispersions Prepared from Phenylenediamine and Optimization of Red Emission. Langmuir 2019, 35, 15257-15266.

(31) Yoshinaga, T.; Akiu, M.; Iso, Y.; Isobe, T. Photoluminescence Properties of L-Cysteine-Derived Carbon Dots Prepared in NonAqueous and Aqueous Solvents. J. Lumin. 2020, 224, 117260.

(32) Ghosh, S.; Ali, H.; Jana, N. R. Water Dispersible Red Fluorescent Carbon Nanoparticles via Carbonization of Resorcinol. ACS Sustainable Chem. Eng. 2019, 7, 12629-12637.

(33) Wang, H.; Sun, C.; Chen, X.; Zhang, Y.; Colvin, V. L.; Rice, Q.; Seo, J.; Feng, S.; Wang, S.; Yu, W. W. Excitation Wavelength Independent Visible Color Emission of Carbon Dots. Nanoscale 2017, 9, 1909-1915.

(34) Zhang, T.; Zhu, J.; Zhai, Y.; Wang, H.; Bai, X.; Dong, B.; Wang, H.; Song, H. A Novel Mechanism for Red Emission Carbon Dots: Hydrogen Bond Dominated Molecular States Emission. Nanoscale 2017, 9, 13042-13051.

(35) Ren, J.; Sun, J.; Sun, X.; Song, R.; Xie, Z.; Zhou, S. Precisely Controlled Up/Down-Conversion Liquid and Solid State Photoluminescence of Carbon Dots. Adv. Opt. Mater. 2018, 6, 1800115.

(36) Reichardt, C. Solvatochromic Dyes as Solvent Polarity Indicators. Chem. Rev. 1994, 94, 2319-2358.

(37) Sato, K.; Sato, R.; Iso, Y.; Isobe, T. Surface Modification Strategy for Fluorescence Solvatochromism of Carbon Dots Prepared from $p$-Phenylenediamine. Chem. Commun. 2020, 56, 2174-2177.

(38) Yaws, C. L. The Yaws Handbook of Physical Properties for Hydrocarbons and Chemicals, 2nd ed.; Elsevier: Amsterdam, 2015; p 84.

(39) Pérez, E. M.; Martín, N. $\pi-\pi$ Interactions in Carbon Nanostructures. Chem. Soc. Rev. 2015, 44, 6425-6433.

(40) Pierson, H. O.; Tonshoff, H. K.; Inasaki, I. Handbook of Carbon, Graphite, Diamonds and Fullerenes: Processing, Properties and Applications; Elsevier: Amsterdam, 1994; pp 44, 45.

(41) Song, Y.; Zhu, C.; Song, J.; Li, H.; Du, D.; Lin, Y. Drug-Derived Bright and Color-Tunable N-Doped Carbon Dots for Cell Imaging and Sensitive Detection of $\mathrm{Fe}^{3+}$ in Living Cells. ACS Appl. Mater. Interfaces 2017, 9, 7399-7405.

(42) Jiang, L.; Ding, H.; Lu, S.; Geng, T.; Xiao, G.; Zou, B.; Bi, H. Photoactivated Fluorescence Enhancement in F,N-Doped Carbon Dots with Piezochromic Behavior. Angew. Chem., Int. Ed. 2020, 59, 9986-9991.

(43) Tan, X.; Li, Y.; Li, X.; Zhou, S.; Fan, L.; Yang, S. Electrochemical Synthesis of Small-Sized Red Fluorescent Graphene Quantum Dots as a Bioimaging Platform. Chem. Commun. 2015, 51, 2544-2546. 
(44) Qu, D.; Sun, Z.; Zheng, M.; Li, J.; Zhang, Y.; Zhang, G.; Zhao, H.; Liu, X.; Xie, Z. Three Colors Emission from S,N Co-Doped Graphene Quantum Dots for Visible Light $\mathrm{H}_{2}$ Production and Bioimaging. Adv. Opt. Mater. 2015, 3, 360-367.

(45) Colthup, N. B.; Daly, L. H.; Wiberley, S. E. Introduction to Infrared and Raman Spectroscopy, 3rd ed.; Elsevier: Amsterdam, 1990; pp 264, 333, 387, 392, 428.

(46) Larkin, P. J. Infrared and Raman Spectroscopy: Principles and Spectral Interpretation, 2nd ed.; Elsevier: Amsterdam, 2017; pp 118, $119,185,186$

(47) Wang, Z.; Yuan, F.; Li, X.; Li, Y.; Zhong, H.; Fan, L.; Yang, S. 53\% Efficient Red Emissive Carbon Quantum Dots for High Color Rendering and Stable Warm White-Light-Emitting Diodes. Adv. Mater. 2017, 29, 1702910.

(48) Silverstein, R. M.; Webster, F. X.; Kiemle, D. J.; Bryce, D. L. Spectrometric Identification of Organic Compounds, 8th ed.; Wiley: Hoboken, 2014; pp 137, 147, 184, 187.

(49) Kondo, Y.; Yoshiura, K.; Kitera, S.; Nishi, H.; Oda, S.; Gotoh, H.; Sasada, Y.; Yanai, M.; Hatakeyama, T. Narrowband Deep-Blue Organic Light-Emitting Diode Featuring an Organoboron-Based Emitter. Nat. Photonics 2019, 13, 678-682.

(50) Yoshinaga, T.; Iso, Y.; Isobe, T. Particulate, Structural, and Optical Properties of D-Glucose-Derived Carbon Dots Synthesized by Microwave-Assisted Hydrothermal Treatment. ECS J. Solid State Sci. Technol. 2018, 7, R3034-R3039.

(51) Yoshinaga, T.; Iso, Y.; Isobe, T. Optimizing the MicrowaveAssisted Hydrothermal Synthesis of Blue-Emitting L-CysteineDerived Carbon Dots. J. Lumin. 2019, 213, 6-14.

(52) Lin, S.; Lin, C.; He, M.; Yuan, R.; Zhang, Y.; Zhou, Y.; Xiang, W.; Liang, X. Solvatochromism of Bright Carbon Dots with Tunable Long-Wavelength Emission from Green to Red and Their Application as Solid-State Materials for Warm WLEDs. RSC Adv. 2017, 7, 4155241560.

(53) Lee, H. J.; Jana, J.; Ngo, Y.-L. T.; Wang, L. L.; Chung, J. S.; Hur, S. H. The Effect of Solvent Polarity on Emission Properties of Carbon Dots and Their Uses in Chlorimetric Sensors for Water and Humidity. Mater. Res. Bull. 2019, 119, 110564.

(54) Pramanik, A.; Biswas, S.; Kumbhakar, P. Solvatochromism in Highly Luminescent Environmental Friendly Carbon Quantum Dots for Sensing Applications: Conversion of Bio-Waste. Spectrochim. Acta, Part A 2018, 191, 498-512.

(55) Basu, N.; Mandal, D. Solvatochromic Response of Carbon Dots: Evidence of Solvent Interaction with Different Types of Emission Centers. J. Phys. Chem. C 2018, 122, 18732-18741.

(56) Yang, L.; Jiang, W.; Qiu, L.; Jiang, X.; Zuo, D.; Wang, D.; Yang, L. One Pot Synthesis of Highly Luminescent Polyethylene Glycol Anchored Carbon Dots Functionalized with a Nuclear Localization Signal Peptide for Cell Nucleus Imaging. Nanoscale 2015, 7, 61046113.

(57) Zhu, S.; Song, Y.; Zhao, X.; Shao, J.; Zhang, J.; Yang, B. The Photoluminescence Mechanism in Carbon Dots (Graphene Quantum Dots, Carbon Nanodots, and Polymer Dots): Current State and Future Perspective. Nano Res. 2015, 8, 355-381. 